[121]

\title{
« Faire passer » : \\ la Russie visible et invisible de Philippe Jaccottet \\ (Après beaucoup d'années)
}

\section{Sur les marges du Hameau}

L'intérêt de Philippe Jaccottet pour la Russie est ancien et profond. Les premières images, nées dans l'enfance à la lecture de Michel Strogoff, se précisent très tôt avec le Livre d'Heures de Rilke issu de ses deux voyages russes ${ }^{1}$. A partir des années 50 , Jaccottet consacre une série d'articles à Alexis Remizov ${ }^{2}$, Boris Pasternak ${ }^{3}$, Marc Chagall ${ }^{4}$, publiés dans la Nouvelle Revue de Lausanne, où il tient la chronique littéraire, et dans la Nouvelle Revue Française. C'est dans la NRL qu'il réagit au procès de Joseph Brodsky ${ }^{5}$, dénonce le "grand théâtre communiste " à propos de celui d'llia Ehrenbourg ${ }^{6}$, ou évoque la difficile parution du Docteur Jivago de Pasternak ${ }^{7}$ dont il traduira plus tard les textes allemands de la correspondance avec Tsvetaieva et Rilke ${ }^{8}$. Mais ce n'est qu'au début des années 70 qu'il fait connaissance avec la poésie de Mandelstam, dans la traduction poétique de JeanClaude Schneider ${ }^{9}$. Il en est tant frappé qu'il veut apprendre la langue du poète russe pour s'en approcher au plus près et, plus tard, la transmettre dans ses propres traductions.

A l'invitation de son ami Pierre Morel, ambassadeur de France en Russie ${ }^{10}$, il visite Moscou et Saint-Pétersbourg. Outre la découverte de la Russie profonde, de la beauté de ses églises et de l'opéra russe (notamment Boris Godounov et le Coq d'or), ce voyage est l'occasion de croiser Soljenitsyne et rencontrer la poétesse Olga Sedakova, rencontre dont la Semaison porte la trace.

C'est pourtant un poème qui n'a pas de lien direct avec la Russie, un poème avec des paysages de montagne très éloignés de ses immensités plates qui servira de fil conducteur à notre

\footnotetext{
${ }^{1}$ Voir Aline Bergé, Philippe Jaccottet, trajectoires et constellations. Lieux, livres, paysages, Lausanne, Editions Payot, 2004. Le livre contient un chapitre particulièrement éclairant sur « L'espace russe » dans la cartographie imaginaire de Philippe Jaccottet, en révélant la signification poétique, onirique, politique et métaphysique.

2 "Du côté de la Russie. Le Veilleur des Misérables", in Philippe Jaccottet, Ecrits pour papier journal. Chroniques 1951-1970, Gallimard, Collection Les Cahiers de la NRF, 1994, p. 77-85. " Le monde du souvenir et le monde du désir : Alexeï Remizov et Louis-Paul Guigues », Nouvelle Revue de Lausanne (par la suite : NRL), 18 juillet 1985.

3 "Quelques notes sur Pasternak », La Gazette de Lausanne, 21-22 mars 1959

${ }^{4}$ "Chagall poète ", NRF, n 209, mars 1970.

5 "Poésie en URSS », NRL, 23 juin 1966 ; « Des poètes à l'Est », NRL, 7 décembre 1968.

${ }^{6}$ "Publications nouvelles à la Guilde du Livre », NRL, 15 décembre, 1961.

${ }^{7}$ " Le Docteur Jivago de Boris Pasternak », NRL, 9 septembre, 1958

${ }^{8}$ Correspondance à trois. Eté 1926, Gallimard, 1983.

9 Publiée dans la revue Argile ( ${ }^{\circ} 7$, été 1975, p. 114-157). Voir à ce sujet l'article d'Alain Paire, "Philippe Jaccottet, lecteur et traducteur de Mandelstam ", http://poezibao.typepad.com/poezibao/2012/04/carteblanche-philippe-jaccottet-lecteur-et-traducteur-de-mandelstam-par-alain-paire.html; Pierre Pachet, «De l'Odyssée à Mandelstam (traductions de poésie) ", in Philippe Jaccottet. Pages retrouvées. Inédits. Entretiens. Dossier critique. Bibliographie. Editions Payot Lausanne, 1989, p. 248-250.

${ }^{10} C^{\prime}$ est sur l'initiative de Pierre Morel que fut édité le premier recueil de poésies de Jaccottet en traduction russe " $B$ свете зимы - A la lumière d'hiver" (sous la rédaction de Nikita Struve, trad. de V. Chvyriaev, A. Davydov), Moscou, Russkij Pout', 1996. Voir sa remarquable préface pour cette édition (Ibid, p. 5-20) et sa version française "Au lecteur russe ", in Revue des Sciences humaines, n²55, juillet-septembre, 1999, p. 131136. Je tiens également à remercier chaleureusement sur le plan personnel Pierre Morel et son épouse Olga grâce à qui j'ai eu la chance et le privilège de rencontrer et m'entretenir avec Philippe Jaccottet.
} 
propos. Intitulé "Hameau ", écrit en 1994 et inclut dans le cycle " après beaucoup d'années ", il nous plonge dans une sorte de vision, entre rêve et souvenir, d'une promenade en montagne sous un col noyé de brume, avec à ses pieds un hameau vide d'habitants. Là, dans ce paysage silencieux et indistinct, le marcheur entend, perçoit plutôt, autrement que par l'ouïe, un appel : « faites passer ».

"Faites passer... »- comme on le fait d'une consigne pour la troupe si le message ne doit pas être ébruité, s'il s'agit d'un secret dont la victoire ou le salut dépend. ${ }^{11}$

La source du message reste mystérieuse : il semble naître de l'air, monter du sol, rumeur silencieuse ; il revient pourtant avec insistance, c'est l'air lui-même qui bourdonne comme un essaim d'abeilles. On pense à ce vers de Mandelstam : " l'air grisâtre est bruissant et moite ${ }^{12}$ ».

Le message vient-il du col ? Peut-être de l'autre côté du col ? D'un autre monde qui s'étend, inconnu, au-delà d'une frontière hermétique, cette Russie dont Jaccottet parle comme d'« une étendue sans limites, plutôt grise, profonde, d'où ne pouvaient [lui] parvenir que des paroles aussi amples et graves qu'elle» ${ }^{13}$. Des paroles qui lui parviennent d'abord par les livres qui sont les vaisseaux de ses voyages: "Toutes ces écritures, toutes ces belles grandes pages, déchiffrables ou non, comme autant de cartes du ciel, comme des signes entre lesquels transparaîtrait un infini » (Ibid, p. 1174).

Ainsi s'ouvrent de nouveaux horizons : les paroles où le poète voyageur croit un moment distinguer "Benedictus qui venit in nomine Domini " $(\mathrm{H}, 835)$, ne viennent-elles pas de plus haut, de plus loin ? Elles n'ont cependant rien de désincarné ; réelles et concrètes, elles sont porteuses de " toute l'épaisseur de la vie ».

Quel est donc ce message ? Peut-on seulement le traduire en paroles ? Qui est le messager ? Ces questions, qui occupent le poète tout au long de sa méditation, font écho aux interrogations et aux expériences des auteurs russes qu'il fréquente.

\section{La transparence des pierres : univers poétique de Mandelstam}

« [...] Autres poèmes de Mandelstam, empruntés à l'anthologie Aubier, et traduits en guise d'exercice matinal. L'inattendu des images: elles surprennent, réveillent; mais leur pouvoir dure. Rien à voir avec l'imagerie surréaliste. Une présence forte du réel. »

Carnets 1968-1979, septembre 1976 (p. 655)

Une première réponse vient avec Mandelstam qui construit toute son œuvre sur le discernement des " signes secrets " présents au cœur de la matière du monde : " les fantômes aussi ont besoin d'un corps, / les paroles aussi participent de la chair ${ }^{14}$.

\footnotetext{
${ }^{11}$ " Hameau », in Philippe Jaccottet, CEuvres, Paris, Gallimard, Bibliothèque de la Pléiade, 2014, p. 834. Toutes les références à l'œuvre de Jaccottet renvoient à cette édition, avec le numéro de page entre parenthèses. Pour « Hameau » : H, p. 834-838.

12 Premier vers du poème « Воздух пасмурный влажен и гулок » (1911), in Mandelstam, Tristia et autres poèmes (trad. de François Kérel), Poésie / Gallimard, 1982, p. 36.

${ }^{13}$ A partir du mot Russie, p. 1175.

${ }^{14}$ « И призраки требуют тела, / И плоти причастны слова ». Роѐте «Как облаком сердце одето» (1910). Notre traduction.
} 
Le poète dont la voix brisée, étouffée, "ressurgit aujourd'hui comme l'eau des torrents " ${ }^{15}$ apparait comme une de ces " ouvertures » entrevues par Jaccottet lors de sa promenade " Au Col de Larche" (évoquée dans le même cycle Après beaucoup d'années) devant, précisément, le « bondissement [...] des eaux alpestres, des eaux glacées » (p. 847), " parole issue, précipitamment, de la bouche de pierre » (p. 850):

Je voudrais faire entendre et, moi-même, écouter sans fin cette parole précipitée, cette voix froide, allègre, sonnant sur des peignes, ou des plectres d'ardoise. Telle qu'il n'en est pas de pareille en ce monde. (p. 851)

Philippe Jaccottet se souvient, en 1981, de sa première lecture quelques années plus tôt du poème de Mandelstam « Je me suis lavé, de nuit, dans la cour » $(1921)^{16}$ :

J'en éprouvai aussitôt l'apparition dans mon espace intérieur, alors presque déserté par la poésie, comme celle d'un météore (dur et brillant), preuve que je n'avais pas tort de continuer à accorder quelque prix à celle-ci, et modèle que je serais d'ailleurs bien en peine de suivre jamais. [...]. Ce qu'évoque ce poème [...] c'est aussi un de ces moments d'une vie qui rendent brusquement aux choses, et justement, en particulier, aux grandes réalités élémentaires usées ou oubliées, leur présence immédiate, leur poids, leurs dimensions presque infinies. ${ }^{17}$

C'est cette densité du réel que rend à son tour l'auteur du Hameau. Comme le tonneau dans la cour qui sert à Mandelstam pour ses ablutions, les objets conservent leur pleine réalité et leur simple fonction pratique : la charrette au bord du chemin "aurait servi encore à transporter du fourrage, du fumier, ou bien on l'aurait laissée se délabrer, mais en aucun cas on ne l'aurait "sauvée" pour y faire trôner des géraniums au milieu d'une pelouse " $(H, 835)$; les fermes sont " vraies » $(H$, 834) comme les « maisons usées qui ont l'air aussi vieillies, aussi vraies que les montagnes » $(H, 835)$.

Ainsi ancrées dans la réalité concrète du monde, les paroles, comme les éléments, retrouvent " vigueur et vérité ". Dans de nouveaux accords entre eux et avec les mots, la terre, le vent, l'eau, le ciel reviennent pour embrasser les cinq sens - faisant tendre l'oreille, attirant le regard, ou pénétrant par le froid. Alors seulement, "franchissant le seuil " sans rien abandonner de "l'épaisseur du temps", nous pouvons "[prendre] racine plus loin que tout cela" $(\mathrm{H}, 837)$. Cet enracinement nécessaire du voyage initiatique est une constante de la poésie de Mandelstam : « moi aussi je quitte l'espace ", écrit-il, mais c'est pour ouvrir le " manuel de l'infini [...] - livre d'énigmes aux énormes racines ${ }^{18}$.

Dans le « Hameau », l'appel à ce voyage est porté par les abeilles :

\footnotetext{
15 "Quelques notes à propos de Mandelstam », Revue de Belles Lettres. Mandelstam, 1981, № 1-4, p. 227-235. p. 227 [Par la suite sous l'abréviation: RBL avec le numéro de la page]. Ces " notes », rédigées à l'occasion de ce numéro spécial, sont reprises dans Une transaction secrète. Lectures de poésie. Gallimard, 1987, p. 172-182, p. 172-3. Nous les citons d'après cette seconde source.

${ }^{16}$ Voir le commentaire de Philippe Jaccottet dans la Semaison - Carnets 1968-1979, note en octobre 1975 (p. 640-641) et dans « Quelques notes à propos de Mandelstam », Op. cit.

${ }^{17}$ "Quelques notes à propos de Mandelstam », Une transaction secrète. Op. cit, p. 172-173. L'auteur revient au commentaire de ce poème dans $A$ partir du mot Russie : «[...] II m'avait frappé comme [...] une chose venue du loin, implacable, mais ressentie en même temps comme une preuve (de ce que la poésie, même aujourd'hui, gardait tout son sens) [...]», Op. cit, p. 1188.

${ }^{18}$ Poème « И я выхожу из пространства ». On trouvera une traduction française par Louis Martinez : « Moi je quitte l'espace », in RBL, p. 105.
} 
Une rumeur, parfaitement silencieuse, un peu plus haut que votre tête. Un froissement sans aucun poids. Des milliers de petites choses éparpillées, à croire qu'il devait y avoir un rucher dans les parages. Et des essaims, pour quelques jours immobilisés dans leur course hésitante, désarmés.

$(H, 836)$.

Le message, silencieux, se condense en rumeur, dans une dualité toute mandelstamienne : audible, il est à peine perceptible ; charnel comme le sont les abeilles ("velues ", écrit Mandelstam), il semble à tout instant prêt à se dissiper : « l'essaim, au premier souffle, sera dispersé, gaspillé, dans un tourbillon (pour faire place, il est vrai, à un autre, de plus en plus opaque, de plus en plus calme) » $(\mathrm{H}, 837)$.

Incarnée dans cette multitude vivante, la rumeur insaisissable s'ouvre à l'expérience de chaque lecteur: "un peu plus haut que votre tête ". L'image se précise dans une nouvelle convergence avec la poésie de Mandelstam où les abeilles se rencontrent fréquemment, notamment dans ce poème traduit par Philippe Jaccottet :

Prends dans mes paumes, pour ta joie, Un peu de soleil et un peu de miel, Les abeilles de Perséphone nous l'enjoignent.

On ne peut détacher la barque non amarrée, $\mathrm{Ni}$ entendre l'ombre chaussée de fourrure, $\mathrm{Ni}$ vaincre, dans la vie épaisse, la peur.

II ne nous reste plus que ces baisers Velus comme les petites abeilles Qui meurent à la porte de la ruche.

Elles bruissent dans les fourrés limpides de la nuit, Leur patrie est l'épaisse forêt du Taygète, Leur aliment : le temps, la bourrache, la menthe.

Prends pour ta joie mon sauvage présent, Ce pauvre collier sec d'abeilles mortes Qui ont transformé le miel en soleil ${ }^{19}$.

Comme souvent chez Mandelstam, les abeilles sont associées à la parole poétique. Le rapprochement, ici, s'ancre dans une source mythologique : les " abeilles de Perséphone ${ }^{20}$ sont les prêtresses de celle qui règne sur les Enfers mais revient, six mois par an, reverdir et revivifier la terre. Ce lien entre mondes visible et invisible, entre le monde concret et charnel des vivants et le monde mystérieux, caché, et pourtant secrètement présent des disparus est aussi la vocation du poète, futce au prix de sa propre vie, à l'image des petites butineuses qui, leur œuvre accomplie, ne sont plus qu'un " pauvre collier sec d'abeilles mortes ». "Elles bruissent » cependant au présent et portent l'image des "baisers velus", traces sonores et sensuelles qui reviennent comme la " matière " principale de l'univers fugace du Hameau.

La ruche, objet concret et familier qui bourdonne dans le Hameau d'une rumeur insaisissable est le mot pivot du poème de Mandelstam. Son seuil, lieu de la transformation du miel en soleil, lieu

\footnotetext{
19 «Возьми на радость из моих ладоней...» in RBL, p. 49 ; « Simple promesse », Op. cit., p. 40.

${ }^{20}$ Nom donné à Eleusis aux prêtresses de Perséphone.
} 
de la naissance de la parole poétique, est aussi celui du passage entre la vie et la mort, souligné dans la traduction de Jaccottet par la mention explicite de la "porte", mot lourd de sens dans le vocabulaire initiatique des deux poètes. On retrouve la ruche dans Autour du mot Russie, comme une image des bibliothèques "avec le foisonnement de leurs milliers de signes, comme d'autant d'abeilles quelquefois fécondes ${ }^{21}$. Elle est aussi, comme chez Mandelstam, le signe d'une révélation personnelle, source de toute poésie, ainsi qu'elle apparaît à plusieurs reprises dans les carnets de Jaccottet intitulés Semaison : "Ce qu'on trouve ainsi par mégarde et qui ne vous troublait plus guère. La ruche. On n'a pas d'âge devant cela. Ou on se l'imagine $»^{22}$.

Le " rucher » du Hameau, porteur des mêmes métaphores, met en lumière la fragilité de la révélation poétique vulnérable au premier coup de vent. Chez Mandelstam déjà, ses petites messagères mouraient sitôt franchi le seuil du lieu mystérieux et secret où se produit l'alchimie merveilleuse de transformation du miel en soleil. Comment n'y aurait-il pas, en effet, hétérogénéité radicale entre ce lieu dont la parole poétique ne récolte que des vestiges (un «pauvre collier d'abeilles mortes ") et notre monde sensible ? Ne faut-il pas " passer outre à ce monde " $(H, 837)$ pour en franchir le seuil ?

«Leur aliment: le temps, la bourrache, la menthe » affirme pourtant Mandelstam à propos des Abeilles de Perséphone ; "toute l'épaisseur du temps, d'une vie, de la vie [...] tout serait sauvé » $(H, 838)$ espère Jaccottet. Le tourbillon ne revient-il pas, " de plus en plus opaque, de plus en plus calme. » $(H, 837)$.

"L'épaisseur de la vie » semble faire écho à la "vie épaisse " de Mandelstam, elle-même mise en parallèle avec "l'épaisse forêt du Taygète " et à la peur qui l'accompagne. Le poète russe, en effet, aura connu, Jaccottet le souligne, tous les cercles de l'enfer dantesque. Le poème "Je me suis lavé, de nuit... ", qui en est comme un pressentiment, est déjà marqué par le froid, celui du premier cercle : une expérience du mal qui peut encore être " changée en bien », un froid qui " dénude et fait briller », reçu par le poète vaudois comme « un baptême brutal »"

Il est remarquable que le "Hameau » est lui aussi marqué par différentes sensations de froid : " un temps encore froid " (836); « il faisait presque froid » (835) ; " par un dimanche froid d'avril » (837). Ce n'est pas, certes, le froid infernal que connaitra Mandelstam mais lui aussi, associé sans doute à la fatigue du chemin, "dénude et fait briller ", comme "l'eau lustrale " dont semble arrosée la nature environnante et qui fait couler "la plus sainte de toutes les eaux », celle des larmes.

Car ce paysage de froid et d'eau, paradoxalement, embrase l'âme d'un feu intérieur :

Dans ce premier cercle, le froid dénude, mais fait briller, il purifie en réduisant toute chose à son noyau ; la terre où nous vivons, rendue par lui plus agressive, n'en devient que plus

\footnotetext{
${ }^{21}$ A partir du mot Russie, Op. cit., p. 1174.

${ }^{22}$ La Semaison, note de juillet 1998, p. 1081. Hervé Ferrage souligne dans la notice aux éditions Pléiade le statut tout à fait exceptionnel de ces Carnets: «Contrairement aux autres livres publiés, qu'ils soit en vers, en prose ou en vers et proses mêlés, ils s'offrent à nous comme des coupes successives, et nécessairement provisoires, dans un processus d'écriture en perpétuel devenir, qui accompagne le passage des saisons et des ans $»($ p. 1408).

${ }^{23}$ "Quelques notes sur Mandelstam », Op. cit, p. 175.
} 
vraie. De pareil heurt, on peut tirer une joie aussi irrécusable que l'étincelle jaillie du silex. $^{24}$

C'est peut-être cet embrasement qu'il s'agit de "faire passer », de transmettre, comme Mandelstam tente de le faire, de la paume à la paume, avec le " miel » et le "soleil " reçus des abeilles. Cette transmission est-elle seulement possible ? Comment dire l'expérience vécue ? Peut-on par la parole, même poétique, "faire passer " la "rumeur silencieuse " ? A défaut, Mandelstam offre au moins son "sauvage cadeau ", ce "collier d'abeilles mortes ", que le traducteur nous transmet à son tour. Ce pauvre vestige de la secrète alchimie, témoignage mort et sec en apparence est, peut-être, secrètement fécond, à la façon de la " pluie sèche de poussière et de pollen ", image associée au tilleul dans la Semaison (autour duquel bourdonnent les abeilles): matière en apparence inerte et sans vie mais qui n'attend que le terreau favorable d'un lecteur attentif et, peut-être, I'humidité de ses larmes, pour croitre et fleurir.

La poésie de Mandelstam, cette " chose venue de loin ", cette "météorite ", entre en résonnance avec celle de Jaccottet et l'éclaire de façon intime. Mais il est d'autre voix, ou plutôt d'autres images qui s'accordent et se mêlent à l'univers de l'auteur du Hameau. Explicitement désignées, ou dessinées sous forme d'ekphrasis, elles apparaissent au détour d'une phrase et participent à déchiffrer le message poétique, comme lors de cette méditation, "Vue sur le lac ", du même cycle Après beaucoup d'année. "Les montagnes de Savoie suspendues au-dessus d'un lac gris comme une masse vraiment énorme qui flottait dans la brume ou dans l'excès de lumière » (p. 813) font d'abord penser au tableau de Magritte Les idées claires, mais aussitôt après c'est Chagall qui " glisse l'idée » d'un jongleur qui jouerait avec l'énorme masse rocheuse (p. 814). Mais ce Chagall « ingénu des années de jeunesse » est ici rapidement congédié.

La première rencontre, virtuelle, de Philippe Jaccottet avec le peintre a lieu à l'occasion de sa traduction de l'allemand de l'imposant Marc Chagall de Franz Meyer ${ }^{25}$, première somme sur l'œuvre de l'artiste, éditée en 1961. Leur dialogue culmine à l'occasion de la traduction, quelques années plus tard, de poèmes de Chagall pour l'édition d'un livre de gravures sur bois ${ }^{26}$ quand, se souvient Jaccottet, il « eu le privilège d'être aidé par Marc Chagall lui-même ${ }^{27}$. S'il insiste sur le fait que leur rencontre est fortuite et n'a eu qu'une incidence mineure sur son œuvre de poète, on peut néanmoins trouver entre les deux artistes des convergences et des résonnances.

\section{« Toute la tristesse de la terre » : transmettre un autre Chagall}

Reprenons le Hameau. La promenade, ou son souvenir, commence comme « un de ces rêves où l'on voudrait que certain nœud moite et vertigineusement doux ne se dénoue jamais " $(H, 834)$.

\footnotetext{
${ }^{24}$ A partir du mot Russie, Op. cit, p. 1189.

${ }^{25}$ Frantz Meyer. Marc Chagall (trad. de l'allemand de Philippe Jaccottet), Flammarion, 1964. Deuxième édition : Flammarion, Paris, 1995. Nous nous référons à la première édition.

${ }^{26}$ Marc Chagall. Poèmes. Gravures originales sur bois de Marc Chagall, Genève, Cramer éditeur, 1968. Traduit par Philippe Jaccottet d'après une version française de Moché Lazar.

${ }^{27}$ Philippe Jaccottet, in Poèmes, Genève, Cramer éditeur, 1975, p. 10. Toutes les citations au livre des Poèmes chagalliens renvoient à cette deuxième édition, préfacée par Philippe Jaccottet.
} 
Un rêve qui ressemble à l'univers chagallien tel que le présente son traducteur : " un monde où l'âme est toujours en mouvement, un monde instable, où elle est sans cesse près de se perdre ${ }^{28}$.

Le Hameau est construit comme une telle errance où une première évocation de la vision, "étrange au point de vous conduire au bord des larmes " $(H, 834)$ revient presque comme une ekphrasis entre parenthèses vers la fin :

" (On imagine encore, justement parce qu'on n'a pas franchi le seuil, lâché son ballot de douleur, qu'il pourrait y avoir ici un afflux, à nos yeux, d'autant de larmes qu'il y a, dehors, de fleurs, brillantes. Toute la tristesse de la terre montée comme de la sève au bord des yeux troublés par l'âge : l'eau la plus sainte de toutes les eaux. Et ce sont elles que l'on croit parfois retrouver éparses dans le ciel, la nuit venue.) » $(H, 837)$

On retrouve l'association des "fleurs brillantes " et des larmes dès la couverture du livre de Frantz Meyer, avec lequel Philippe Jaccottet passe plusieurs mois. L'image reprise dans la première édition française, montre un autoportrait de 1944 de Chagall penché sur sa toile devant un bouquet de fleurs, des larmes dessinant comme un personnage sur sa joue (Figure 1). On retrouve cet accord au milieu du livre, dans une nouvelle composition intitulée "Les poissons de Saint Jean ». Comme dans le Hameau, un seuil est suggéré : le regard du spectateur est attiré, à la suite des poissons du titre du tableau, vers

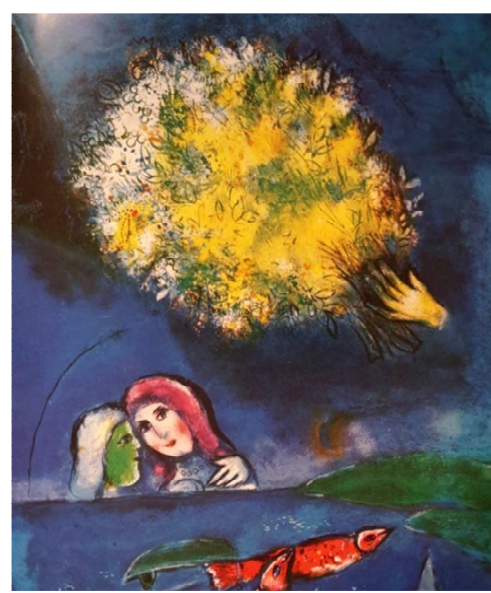

Figure 2. Marc Chagall. Les poissons de Saint Jean, in Frantz Meyer, Marc Chagall (1961), p. 499.
I'au-delà de l'horizon où nous appelle le regard du personnage central et audessus duquel flotte un immense bouquet tel un astre brillant. Les larmes, d'émotion, sont suggérées par le couple réuni, enlacé, audelà du "seuil ", et il est permis de penser que ce sont elles qui remplissent le fleuve ${ }^{29}$, large métaphore de l'existence humaine dans ses tempêtes et ses temps de paix.

« Laisser mes yeux pleurer sans cesse / Les larmes s'ajouter au fleuve ", écrit en effet Chagall, traduit par Jaccottet, dans le poème «Comme dans un dernier sommeil» ${ }^{30}$. Le traducteur est sensible à cette tonalité de l'œuvre du peintre qui, à de rares exceptions comme Yves Bonnefoy ${ }^{31}$, échappe à ses compatriotes qui voient dans la peinture de Chagall un univers féérique (Aragon), surnaturel (Breton), acrobatique (Eluard), amoureux (Marcel

\footnotetext{
${ }^{28}$ Philippe Jaccottet, Préface, Ibid, p. 9.

${ }^{29}$ On lit, par exemple, dans le poème " Des chemins »: " Mes larmes tombent comme des cailloux / Elles se mêlent au fleuve / Elles flottent comme des fleurs / Ainsi je vis, mon Dieu. [...] », Op. cit, p. 65.

${ }^{30}$ Op. cit, p. 89.

${ }^{31}$ Voir la méditation d’Yves Bonnefoy "La religion de Chagall ", Derrière le Miroir, 1962, n 132, p. 1-19. Nous l'analysons dans l'article " "Le retour possible à la condition de Lumière" : dialogue entre Yves Bonnefoy et Anna Akhmatova autour de l'œuvre de Chagall ", in Yves Bonnefoy. Poésie et dialogue. Textes réunis par Michèle Finck et Patrick Werly. Strasbourg, PUS, 2013, pp. 371-392.
} 
Arland $)^{32}$. C'est ce Chagall-ci qui suggère l'idée de ce passant sur le quai, jonglant avec les montagnes, qui semble échappé des décors pour le Théâtre Juif créé par le peintre au début des années 20, et que le poète a vu et apprécié au Musée de la Fondation Pierre Gianadda à Martigny où ils étaient exposés au milieu des ruines romaines. Sur ces panneaux, l'univers tout entier s'anime sur un rythme d'allegro, entrainé par les musiciens, les acrobates et les acteurs. Pourtant, déjà, transparaît une profonde mélancolie, voire la tragédie intérieure du peintre si lumineux et joyeux aux yeux de tous. C'est le Chagall qui sera l'interlocuteur privilégié du poète. Celui-ci le cite après leur rencontre à Saint-Paul-de-Vence en 1966 : "Charge affective des mots: d'autant plus grande probablement qu'elle est plus cachée. Ce que Chagall m'a dit de Mozart : plus sa musique est transparente, plus la mort y est sensible. Je ne suis pas sûr que ce soit exact, mais il y a une vérité et une beauté dans cette pensée $[\ldots] »^{33}$.

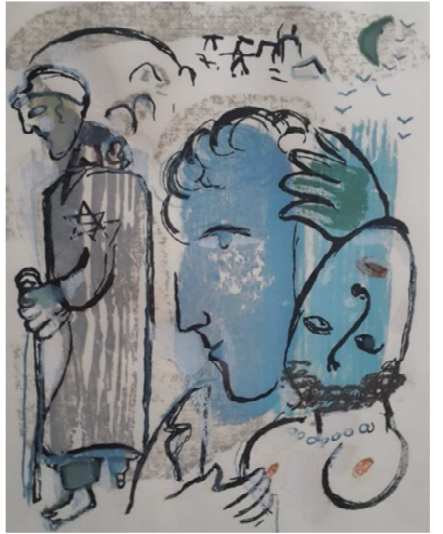

Figure 3. Epreuve en bois pour l'édition des Poèmes de Chagall (1968). Collection personnelle de Philippe Jaccottet

Chagall porte en lui une nostalgie, inséparable de la tragédie de son peuple exilé depuis "deux mille années " ${ }^{34}$. Une gravure en bois réalisée pour l'édition de ses Poèmes et conservée par Philippe Jaccottet sur un mur de sa maison en témoigne (Figure 3). On y voit la figure du vieux juif errant accompagnée de celle du peintre sous les traits d'Orphée dont la lyre chante les vestiges de leur passé, et porte leur espoir qui demeure comme une grande tache bleue au milieu du tableau.

Cette nostalgie n'est pas étrangère à l'auteur du Hameau. Une " semaison de larmes " irrigue sa poésie depuis Airs, se fait amère dans Obscurité (1961), avant de devenir épiphanique quand "l'afflux » de larmes, "la plus sainte de toutes les eaux ", fait fleurir les fleurs des champs et briller les étoiles du ciel $(H, 837)$.

L'enjeu principal de la poésie chagallienne reste cet instant épiphanique qui permet d'entrevoir une plénitude de la vie que Philippe Jaccottet retrouve dans l'œuvre de Hölderlin, Rilke ou Mandelstam. Chagall, qui aide le poète à le traduire, insiste, raconte celui-ci, pour garder une parole "simple ", voire "sauvage", pour refuser " les articulations trop logiques (qui nous échappent presque inconsciemment, à nous autres occidentaux "raisonnables") ${ }^{35}$. C'est cette résistance des écrivains russes à toute sorte de " démonstrations », " abstractions » ou " dogmatismes » que Jaccottet apprécie ${ }^{36}$, lui dont la quête poétique vise à libérer l'expérience immédiate du filtre réducteur des concepts ${ }^{37}$.

Cette expérience, pour Chagall, est d'abord celle d'une présence: "je sais qu'il y a des hommes comme des fleurs qui me consolent, de loin, de quelque part ", écrit-il dans une lettre

\footnotetext{
${ }^{32}$ Voir la revue Derrière le miroir qui a consacré dans les années 60 plusieurs numéros à l'œuvre chagallienne à l'occasion de ses grandes expositions en France.

${ }^{33}$ La Semaison, Carnets 1954-1967, note en juin 1966, p. 397.

34 "Toutes ces années à compter des larmes / J'ai cherché sous le ciel et sur la terre / Deux mille années j'ai attendu / que mon cœur s'apaise [...] », "Sur le pays neuf » in Marc Chagall. Poèmes, Op. cit, p. 85-86.

${ }^{35}$ Philippe Jaccottet, "Préface", Ibid, p. 11.

${ }^{36}$ " De Jules verne à Leskov », NRL, 14 septembre, 1951 ; « Le Docteur Jivago de Boris Pasternak », NRL, 9 septembre, 1958.

37 « Poésie : mieux on comprend comment cela devrait se faire, moins on y parvient. La virtuosité apparait avec le vide », La Semaison, note en mai 1971, p. 599.
} 
publiée dans l'ouvrage de Meyer pour accompagner les Poissons de Saint Jean ${ }^{38}$. L'auteur de "Paysages avec figures absentes " ne peut être insensible à cette présence qui perce à travers les interstices, lui qui en esquisse dans le Hameau, notamment avec cette scène de fête de jeunes filles: " Elles-mêmes ont dû s'en aller ; ne persiste plus que leur image enfuie, leur absence, parfumée " ( $H$, 835). Comme dans l'exemple des poissons de Saint Jean, elle s'exprime souvent chez Chagall à travers des images bibliques qui imprègnent l'univers intérieur du peintre juif russe. Ces images sont plus rares, presque absentes, chez le poète sans doute parce que, trop usées, elles videraient la réalité vécue de sa substance pour la faire apparaitre " diaphane, spectrale, glacée ", très loin de la tendresse charnelle qui habite la peinture de Chagall et de la densité du réel que Mandelstam ou Jaccottet explorent dans leur poésie. Elles sont pourtant évoquées, en creux, par la mention répétée que la promenade a lieu un dimanche, plus explicitement par la reprise de l'exclamation du psalmiste Benedictus qui venit in nomine Domini - étonnement émerveillé devant la Présence qui ne se cristallise pas encore dans les mots de la théologie ou de l'interprétation conceptuelle, comme ce dimanche "sans aucune cloche, sans prêche, sans paroisse ». On songe au "murmure doux et léger » dans lequel l’Eternel apparaît au prophète (1 Rois 9,12).

Le même sentiment naît de la lecture par le poète de la "Nuit de Pâques " de Tchekhov, où la " présence devinée d'une foule heureuse ${ }^{39}$ accompagne la traversée du fleuve vers le lieu de la fête, traversée semblable à ce moment où « l'on passe un seuil, [et où] commencent, peut-être, les visions " $(H, 835)$. C'est cette même faculté de glisser du visible à l'invisible, déjà notée dans la poésie de Brodsky en $1966^{40}$ qui attire Philippe Jaccottet dans celle d'Olga Sedakova dont il fait la connaissance en Russie en mai 1996. Tous deux partagent un fort attachement pour l'Italie, Ravenne en particulier, et dialoguent dans la langue de Dante.

\section{«Seule l'absence regarde. Seul l'invisible voit » : le dialogue avec Olga Sedakova}

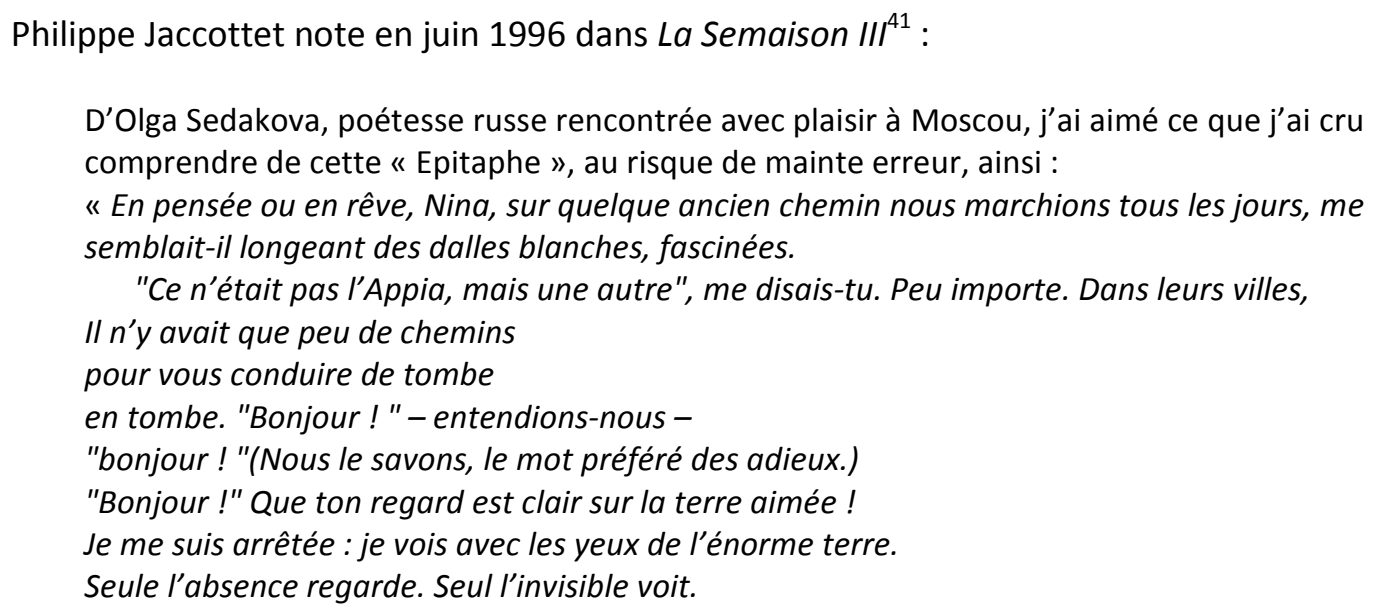

\footnotetext{
${ }^{38}$ Frantz Meyer. Marc Chagall, Op. cit, p. 496.

${ }^{39}$ A partir du mot Russie, p. 1175.

40 "Poésie en URSS », NRL, 23 juin 1966.

${ }^{41}$ Première publication : Carnets 1995-1998 (La Semaison III), Gallimard, collection « Blanche », 2001
} 
Presse donc le pas : je te dépasserai. " (p. 1030)

Ce poème, tiré du recueil "Stèles et inscriptions " ${ }^{42}$ (1982), fait partie d'un cycle intitulé "Après avoir soigneusement étudié ce sujet" et dédicacé à Nina Braginskaia, spécialiste de l'ekphrasis antique, avec qui la poétesse partage sa promenade imaginaire. Elle y met en scène six monuments antiques et entre en dialogue avec leur histoire.

Sachant qu'il lit le russe et qu'il a traduit Mandelstam, Olga Sedakova offre ce poème à Philippe Jaccottet qui l'inclut dans sa Semaison, ce « recueil de graines légères, [...] des clartés reçues non seulement du monde mais aussi d'écrivains fort divers comme La Fontaine, Goethe, Maurice de Guérin, Claudel [...] $»^{43}$.

Sa traduction donne à entendre au lecteur francophone cette nouvelle voix venue " de l'autre côté du col » qui plonge dans le souvenir de la Rome antique pour une promenade " en pensée ou en rêve ". Le titre, "Epitaphe ", met l'accent sur le message gravé sur les tombes, témoins d'un passé lointain à la manière des "fermes de très vieille pierre et de très vieux bois " du Hameau. Tout comme ce dernier, c'est le lieu même, vidé de ses habitants, qui se met à parler : "Bonjour ! "entendions-nous -», allusion au mot initial des inscriptions sur les tombes antiques ("Bonjour! Réjouis-toi ! " $)^{44}$ qui ainsi "parlent " au sens presque littéral. II ne s'agit pourtant pas de voix d' " outre-tombe " qui monteraient de sous la terre mais d'une voix portée par la terre elle-même, comme celle qui guide le poète du Hameau et le retient de "prendre congé » de ce monde $(H, 837)$, de cette " terre aimée » selon l'heureuse formule de Philippe Jaccottet. L'expression russe, «милая земля» (terre "douce ", " chère »), exprime en effet un profond attachement à la terre que l'on retrouve chez la poétesse, avec les mêmes mots, dans le Voyage en Chine ${ }^{45}$ où perce le souvenir du Voyage en Arménie de Mandelstam. Philippe Jaccottet accentue cet élan vers la terre en ajoutant un point d'exclamation, lui qui tient sa propre lyre au plus près de la terre. II va dans sa traduction jusqu'à identifier le moi lyrique avec celle-ci : " je vois avec les yeux de l'énorme terre ». Cet écart, peut-être involontaire, du sens littéral (" je regarde avec des yeux plus énormes que la terre »), est cependant conforme à l'intention profonde de la poétesse : il ne s'agit pas dans ces vers de se placer

\footnotetext{
${ }^{42}$ Olga Sedakova, Стелы и надписи, Санкт-Петербург, изд. Ивана Лимбаха, 2014, р. 19. Нина, во сне ли, в уме ли, какой-то старинной дорогой шли мы однажды, как мне показалось, вдоль многих белых, сглаженных плит. - Не Аппиева, так другая - 
au-delà de la terre (à laquelle toute son œuvre chante un hymne), c'est une façon de rendre la mesure cosmique et intemporelle du lieu et de l'instant où l'écrivain est saisi par la poésie qui ne peut être comparé qu'avec la terre, concrète et démesurée, "énorme " comme les racines de I'univers de Mandelstam.

Chez Sedakova, ce saisissement passe d'abord par le regard. Non pas pour percer "l'invisible ", car l'invisible est le sujet, mais pour être vu, percé par lui : "Seule l'absence regarde. Seul l'invisible voit ». Chez Jaccottet c'est le silence qui parle et appelle : " "Faites passer", disait la terre elle-même, ce matin-là, de sa voix qui n'en est pas une " $(H, 837)$. C'est, faisant jouer des sens différents, un même mystère qui interpelle le poète. Mystère discret, prêt à disparaître au moindre mouvement de l'air, mais qui enveloppe et submerge celui qui accepte d'y prêter attention.

Les deux poètes sont de ceux-là qui choisissent de répondre à cet appel, "franchir le seuil ", pour avancer dans l'inconnu " sans trop chercher à comprendre " (Jaccottet), et sans se soucier de la route qu'ils prennent : « peu importe » (Sedakova).

Quand, à son tour, Olga Sedakova transmet au lecteur russe les fruits de leur rencontre en traduisant des poèmes du livre I'Air $^{46}$, son choix de textes est significatif en ce qu'il touche à son propre univers poétique tissé de vent et du mouvement des arbres, à travers lesquels murmure " une voix sourde qui parle de jardins extraordinaires ${ }^{47}$.

Elle apprécie particulièrement chez Jaccottet son effort pour « supprimer radicalement du vocabulaire - et, surtout, de l'état d'esprit même de l'auteur, tout ce qui n'est pas authentique ${ }^{48}$. C'est cette authenticité, que tous deux partagent avec Mandelstam ou Chagall, qui permet au bout du compte d'affirmer :

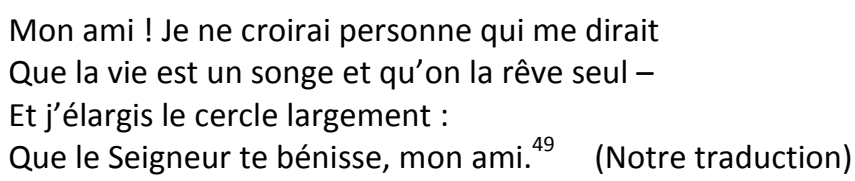

Philippe Jaccottet élargis à son tour son cercle d'amis poètes dans le livre Une transaction secrète dont le titre renvoie à l'Orlando de Virginia Woolf : "Ecrire de la poésie, n'est-ce pas une transaction secrète, une voix répondant à une autre voix ? $\|^{50}$. Des voix du monde entier sont inclues dans cette polyphonie, dont plusieurs (Hölderlin, Rilke, Celan ...) sont celles-là même qu'Olga Sedakova « fait passer » au lecteur russe par ses traductions.

La flûte répond à la flûte, Non celle faite d'os, ni de bois, Mais celle que les montagnes

\footnotetext{
${ }^{46}$ Choix de poèmes publié en russe sous forme de recueil sous le titre В комнатах садов - Dans les chambres des vergers, Арт - Волхонка, 2014.

47 « голосом, говорящим глухо про небывалые сады», Роѐmе « Может, ты перстень духа» du cycle Kитайское путешествие (Voyage en Chine), Op. cit, p. 18, http://polutona.ru/?show=1017164025.

${ }^{48}$ "Simple profondeur. Notes du traducteur», Dans les chambres des vergers, Op. cit, p. 89.

${ }^{49}$ Стансы в манере Александра Попа (Stances à la manière d'Alexandre Pope), 4, http://www.vavilon.ru/texts/sedakova1-07.html

Мой друг! Я не поверю никому, что жизнь есть сон и снится одному и я свободно размыкаю круг: благослови тебя Господь, мой друг.

${ }^{50}$ Philippe Jaccottet, « Une transaction secrète », Gallimard, 1987, p. 9. La première mention de la citation de Virginia Woolf dans La Semaison, juillet, 1991, p. 876.
} 
Gardent dans leurs grottes et leurs failles. ${ }^{51}$ (Notre traduction)

Nombre de ses compatriotes participent naturellement à cette "transaction secrète " circulaire. On peut entendre ici Mandelstam qui sait « lier avec la flûte " les espaces et les temps, ou le roseau d'Anna Akhmatova qui "renait et chante " ${ }^{52}$ et fait vivre "le chœur secret " des amis disparus ${ }^{53}$. Philippe Jaccottet entend à son tour, en 1967, ce chant qui " échappe aux doctrines politiques comme aux modes esthétiques ${ }^{54}$. Une voix en entraine une autre, comme on le devine dans une lettre, un an plus tard, qui marque le passage d'A la lumière d'hiver au "livre apaisé $\|^{55}$ qu'est Pensées sous les nuages:

Chaque matin, je déchiffre un poème russe, à grands coups de dictionnaire; et cette poésie si chaleureuse (de Blok, d'Akhmatova, de Mandelstam, de Tsvetaieva) me console du dessèchement progressif de la récente poésie française - et m'aide à retravailler moi-même. ${ }^{56}$

Ces voix lointaines, qui permettent un renouvellement de son art et de soi-même, recherchent elles-mêmes leur lecteur sous des horizons inconnus. Mandelstam souligne le paradoxe dans son essai l'Interlocuteur : la poésie est dialogue mais l'interlocuteur du poète est le lecteur de hasard, providentiel, inconnu de lui. Seule la distance fait naitre le désir et la possibilité du dialogue en profondeur. " Si je connais mon interlocuteur je connaitrai par avance ses réactions aux paroles que je lui adresse. Comment m'étonner alors de son étonnement, me réjouir de sa joie ou aimer son amour? ". Cet interlocuteur inconnu, "dans l'existence duquel le poète doit croire, sous peine de douter de lui-même ${ }^{57}$, est peut-être alors celui qui, " de l'autre côté du col », déchiffre patiemment les mots d'une langue inconnue.

\section{A partir du mot Russie, le mot Esperance}

\section{Quel message, quelle consigne se découvre ainsi peu à peu ?}

Il est deviné dans les mots de Mandelstam, dans les images de Chagall, au rythme des pas d'Olga Sedakova; il est inséparable du chemin parcouru, du contact avec la terre. Sur ce chemin, toujours secret et personnel, les poètes se croisent parfois, se rencontrent, font un bout de chemin ensemble, tous sensibles à la beauté discrète du monde qu'ils nous proposent de contempler. Tous prennent le temps de s'arrêter devant l'instant où la poésie " se noue et se dénoue ${ }^{58}$, celui où l'on se tient en haut du col, " un de ces moments d'une vie qui rendent brusquement aux choses [...] aux

\footnotetext{
${ }^{51}$ Du cycle Kumaйское путешествие (Voyage en Chine), Op. cit, p. 20.

Флейте отвечает флейта,

не костяная, не деревянная,

а та, которую держат горы

в своих пещерах и щелях,

струнам отвечают такие же струны

и слову слово отвечает

52 Akhmatova, Anna. "Le Roseau - Тростник». Voir la trad. de Jean-Louis Backès, Poésie - Gallimard, p. 169.

${ }^{53}$ Akhmatova, Anna. "Poème sans héros - Поэма без героя», Ibid, 234.

54 "Poètes en URSS ", NRL, 23 juin 1966. La traduction du Requiem cette même année par Paul Valet aux éd. Minuit a marqué cette génération. Voir par exemple le témoignage d’Yves Bonnefoy "Oui, je le peux ", in Anna Akhmatova et la poésie européenne, Bruxelles, Peter Lang, 2016, p. 241-242.

55 José-Flore Tappy, Notice pour Pensées sous les nuages, in Jaccottet, đuuvres, Op. cit., p. 1487.

${ }^{56}$ septembre 1976. Notice pour Pensées sous les nuages, p. 1486

${ }^{57}$ Mandelstam, «O собеседнике», Собрание сочинений в четырех томах, Moskva Terra, 1991, vol. II, p. 239-240. Notre traduction.

${ }^{58}$ Mandelstam, «Может быть, это точка безумия» (1937), in Собрание сочинений в четырех томах, Ор. cit, vol. I, p. 258.
} 
grandes réalités élémentaires usées ou oubliées, leur présence immédiate, leur poids, leurs dimensions presque infinies $1{ }^{59}$. Ce moment suspendu, comme hors du temps, est celui qui redonne vie et consistance au temps :

Toute couleur, toute vie naît d'où le regard s'arrête ${ }^{60}$

Chagall le représente comme une horloge suspendue, Olga Sedakova le rend par la simplicité enfantine et la sonorité cristalline de sa poésie ou encore en traduisant en russe le vers ci-dessus ${ }^{61}$.

La disponibilité des auteurs russes à l'étonnement, la faculté de transmettre, au présent, la joie de la naissance du monde, sont pour leur interlocuteur vaudois, le signe d'une grande sincérité et authenticité, la manifestation la plus sûre de la Beauté.

Mais celui-ci connait aussi, avec Mandelstam, "la beauté des choses qui blessent " ${ }^{62}$ et mesure, avec Dostoïevski, le prix de cette Beauté, conservant jusqu'à aujourd'hui dans sa bibliothèque $L a$ voix souterraine, acheté à l'âge de 20 ans $^{63}$ et portant, contrairement à ses habitudes, de multiples marques au crayon sur les marges. "C'est que ce livre est vraiment notre $D e$ Profundis; mais le cri en est pire, parce qu'il ne s'adresse plus à personne et parce que celui qui crie ne sait plus s'il est encore quelqu'un ; parce qu'il se déchire, se retourne contre lui-même et n'a plus d'autre certitude que celle du mur contre lequel il bute. (Job, XIX, 81, déjà : "il a dressé sur ma route un mur infranchissable")..1 ${ }^{64}$

Ce mur est ébranlé pourtant chez ce même Dostoïevski par le rire "limpide et frais » d'une jeune fille ou "le mélange de tendresse et de gaité » qui saisit Aliocha Karamazov à la lecture de la lettre de son amie Lise ${ }^{65}$.

Philippe Jaccottet confie, dans un entretien récent ${ }^{66}$, son intuition que la poésie de l'Est touche quelque chose de profondément humain, qu'elle a un sens de la douleur qu'elle connait d'expérience, et quelle sait la traduire au mieux. A la suite des auteurs russes, il visite ces lieux où notre siècle fait "monter à la surface du monde du monde réel, avec des hommes réels ", la vision de Dante. Mais de même que celui-ci est guidé par Virgile vers une issue, de même ceux-là lui indiquent une voie " pour revenir au monde clair ». Le poète Varlam Chalamov est l'un de ces guides, lui qui a connu les camps staliniens de la Kolyma :

Le froid, là, quand Chalamov le mesure, est encore d'une dizaine de degrés plus intense ; mais ce n'est pas le pire. A la dureté du froid s'ajoutent ici la faim, l'épuisement par la faim, les maladies, le travail forcé, les coups. Je ne veux pas, je ne pourrais pas répéter ces récits.

Mais Jaccottet ajoute, dans le paragraphe suivant :

Or, malgré cela, Chalamov survit; non seulement survit, mais revient à la vie avec une foi inentamée en cette parole de la poésie dont il assure qu'elle sera restée, à travers le pire, sa «forteresse ». Comment ne pas faire confiance à pareil témoin ? ${ }^{67}$

\footnotetext{
59 "Quelques notes sur Mandelstam », Op. cit, p. 173.

${ }^{60}$ Philippe Jaccottet, "Toute fleur n'est que de la nuit », cycle " Oiseaux, fleurs et fruits », Airs, p. 425.

${ }^{61}$ В комнатах садов - Dans les chambres des vergers, Op. cit, p. 18.

62 Expression de Pierre Pachet, « De l'Odyssée à Mandelstam », Op. cit, p. 250.

${ }^{63}$ Fédor Dostoïevsky, La voix souterraine (traduit du russe par Boris de Schloezer), Librairie Stock, 1926.

${ }^{64}$ A partir du mot Russie, p. 1179-1180. Voir, sur Dostoïevski et Jaccottet, les réflexions de Arina Kouznetsova, "Jaccottet en Russie ", in Europe n`955-956, 2008, numéro spécial consacré à Philippe Jaccottet / Giuseppe Ungaretti, p. 190-191 ; Aline Bergé, "Mélancolie et félicité de Dostoïevski », in Philippe Jaccottet, trajectoires et constellations, Op. cit, p. 336-338.

${ }^{65}$ A partir du mot Russie, p. 1186.

${ }^{66}$ Entretien le 16 février 2015 avec Tatiana Victoroff.

${ }^{67}$ A partir du mot Russie, p. 1191.
} 
C'est ainsi que renait le mot que, selon le poète florentin, il fallait laisser à la porte de l'Enfer mais qui, dans la lecture de Jaccottet s'esquisse à nouveau dans les derniers vers de la Divine Comédie. Il brille, comme une étoile dans l'obscurité, au firmament de la littérature russe ; c'est lui que l'on discerne dans l'essaim de Mandelstam, au milieu des fleurs et des larmes de Chagall, et qui prend chair du "Bonjour! " qui s'élève des tombes de l'antiquité. Ce mot Espérance, se dessine comme la substance du " secret dont la victoire ou le salut dépend » $(H, 834)$.

On entend en filigrane la voix d'Anna Akhmatova, cet autre témoin de l'espérance impossible qu'elle entend "chanter" dans les moments les plus étouffants du Requiem ou celle de Nadejda Mandelstam dont le livre de souvenirs "Contre tout espoir » fut la première rencontre de Jaccottet avec Ossip.

"La métaphysique n'est pas en cause. La réalité seule peut appeler à la vie une réalité nouvelle ", écrit Mandelstam dans L'interlocuteur. C'est cette espérance qui embrasse toute la réalité de ce monde, jusqu'en ses tréfonds les plus obscurs que Jaccottet retrouve chez les auteurs russes, de Dostoïevski et Tolstoï à Mandelstam et Pasternak ${ }^{68}$, et c'est ce caractère concret qui donne sa plénitude et sa force au mot espoir qui apparait à l'horizon du Hameau. Un autre mot encore est suggéré par le vocabulaire quasi liturgique ("tout serait sauvé »); il n'est pas prononcé ici par Jaccottet - ce vocabulaire n'est pas le sien - mais est incontournable dans l'article $A$ partir du mot Russie $^{69}$ : celui de Résurrection.

Il est significatif que Jaccottet choisisse pour parler de ce thème vital de la littérature russe des images charnelles qui rendent la puissance du réel : la Résurrection est comparée, avec Tchekhov, au moment où "le fleuve rejette ses chaînes de gel, [fait] craquer les coutures de sa cuirasse, enfle la voix comme les orgues pour célébrer la force du printemps " (A partir du mot Russie, p. 1176). C'est encore la puissance de l'eau qu'évoque le rire d'Aglaé chez Dostoïevski :" [...] non pas angélique, non pas désincarné, rien dans le monde naturel ne lui ressemble plus que le torrent : quelque chose a bondi à votre rencontre [...] pour vous abreuver ou vous laver, [...] une brèche s'est ouverte dans le mur de pierre contre lequel le crâne allait, à sa longue, se briser, cette eau jaillissante vous dispense de toute autre clef ${ }^{70}$. Rien de plus réel et de concret que la puissance du torrent dévalant des montagnes, une réalité libre et vivante qui heurte et brise cette autre réalité, figée et close, du mur qui semblait inébranlable.

Jaccottet conclut ainsi sa longue méditation sur le mot « Résurrection » dans $A$ partir du mot Russie :

" Je me demande si l'homme que je suis, né et élevé dans ce monde-là, n'aurait pu croire cette parole et la répéter avec la même joie, en dépit de toute raison, puisqu'elle n'était pas moins absurde alors qu'aujourd'hui où je l'entends dans une langue vraiment étrangère et imprononçable. A croire qu'il y aurait, même pour les vérités proclamées "éternelles", des lieux et des moments privilégiés » (p. 11761177)

Ne peut-on voir dans le Hameau, un tel lieu, un tel moment ? Le mot n'est pas prononcé : sans doute risquerait-il d'être " dispersé, gaspillé » $(H, 837)$ dans le tourbillon des mots trop usés, épuisés par le discours des théologiens. Pourtant certains d'entre eux n'y reconnaitraient-ils pas leur "plus haute et secrète espérance ${ }^{71}$ ? On songe à Karl Barth, qui apparait dans les écrits de

\footnotetext{
${ }^{68}$ "Une vague de roman ", NRL, 24 octobre 1958.

${ }^{69}$ Voir notamment l'analyse de La Nuit de Pâques de Tchekhov, in A partir du mot Russie, p. 1175.

${ }^{70}$ A partir du mot Russie, p. 1187. Cette relecture de l'Idiot remonte à novembre 1990, voir la note dans $L a$ Semaison, Carnets 1980-1994: "[...] Le "rire claire et frais" d’Aglaé qui éclate après une succession de cauchemars est, rigoureusement l'irruption du torrent », p. 943.

${ }^{71}$ A partir du mot Russie, p. 1176.
} 
Jaccottet à propos de la Passion de Bach, ou à Olivier Clément, penseur français converti au christianisme après sa rencontre avec l'orthodoxie russe, et qui, pour parler de la Résurrection, se fait volontiers poète. Quand Clément, grand lecteur des auteurs russes, de Dostoïevski à Chestov, commente avec force ce passage de Crime et Châtiment ou l'assassin et la prostituée lisent ensemble, chez Saint Jean, le récit de la résurrection de Lazare, il pourrait, sans en changer une parole, exprimer avec les mots de Jaccottet la foi du romancier russe : " toute l'épaisseur du temps, d'une vie, de la vie, avec leur pesanteur, leur obscurité, leurs déchirures, leurs déchirements, tout serait sauvé » $(H, 838)$.

\section{Des messagers venus de L'Est}

« Faites passer », disait la voix discernée par le poète du Hameau venant du col.

" Faites passer " c'est peut-être ce qu'ont entendu aussi les poètes et les écrivains russes lus et traduits par Philippe Jaccottet. Porteurs d'une autre culture et d'une langue "vraiment étrangère ", d'une expérience historique douloureuse mais aussi révélatrice, eux aussi perçoivent et déchiffrent des bribes de ce message silencieux.

L'appel secret et mystérieux s'éclaircit un peu, par bribes, avec le concours de ces messagers venus de l'Est. "Simple promesse ${ }^{72}$ ou " pensées de voyageur, pour peu que l'enveloppe un manteau de grésil ", la formulation reste prudente, les pas sont hésitants, mais le chemin parcouru ensemble permet de dire parfois, traduisant Mandelstam : " Je ressusciterai pour dire que brille le Soleil ! ${ }^{73}$.

Par-delà les frontières et les langues, par-delà même les différences de sensibilité et d'appréhension du monde, mais dans une même disponibilité et ouverture à la réalité la plus concrète telle qu'elle nous apparait dans ces instants " plus fugaces que le passage du vent ${ }^{74}$ et qui sont selon Yves Bonnefoy, l'origine de toute poésie ${ }^{75}$, la rencontre et le dialogue entre les poètes devient mystérieusement possible. Alors ils se font à leur tour les messagers, nous transmettent, de façon un peu plus claire, un peu plus audible, plus accessible, la consigne " faites passer... " à travers ce « livre idéal » qu'imagine Philippe Jaccottet :

«Le livre idéal n'est pas le recueil des poèmes; il n'en comporterait qu'à ses moments les plus purs, comme des fêtes dans l'année verbale [...] Mais ce livre idéal se compose, en fait, de plusieurs livres d'auteurs différents, chacun n'en pouvant réaliser que certains aspects, n'en écrire que certaines pages. »

La Semaison, [octobre 1966], p. 402

\footnotetext{
72 Les derniers mots du poème "Sur la terre vide » (le 4 mai 1937, Voronèje), in RBL, p. 138; "Simple promesse ", Op. cit, p. 143.

73 Le dernier vers du poème « Des tertres de têtes humaines s'étendent au loin » (1937), in RBL, p. 116; «Simple promesse », Op. cit, 143.

${ }^{74}$ Philippe Jaccottet, "Images plus fugaces » in « Oiseaux, fleurs et fruits », Airs, p. 432.

${ }^{75}$ Yves Bonnefoy, "La traduction de la poésie ", in L'autre langue à portée de voix, La librairie du XXI siècle / Seuil, p. 32 et suivantes.
} 
